\title{
Eine Real-World-Studie bei Vorhofflimmern
}

Fragestellung: Wie sind Wirksamkeit und Blutungskomplikationen bei einer oralen Antikoagulation bei neu diagnostizierten Patienten mit nicht valvulärem Vorhofflimmern, wenn Dabigatran und Warfarin verglichen werden?

Hintergrund: Vor fünf Jahren wurden die Ergebnisse der RE-LY-Studie vorgestellt, die zwei Dosierungen von Dabigatran $(2 \times 150$ und $2 \times 110 \mathrm{mg})$ mit Warfarin bei Patienten mit nicht valvulärem Vorhofflimmern verglich [1]. Sie zeigte, dass die höhere Dosis von Dabigatran bei einem vergleichbarem Blutungsrisiko bezüglich der Schlaganfallprävention Warfarin überlegen war. Die niedrigere Dosis war genauso wirksam wie Warfarin, aber mit niedrigerem Blutungsrisiko. Patienten, die in randomisierte Studien eingeschlossen werden, sind allerdings hoch selektioniert und nicht unbedingt repräsentativ für Patienten, die nach der Zulassung eines Medikamentes behandelt werden. Daher ist es sehr wichtig, Wirksamkeit und Blutungskomplikationen im klinischen Alltag zu untersuchen.

Patienten und Methodik: Die Studie stützt sich auf die Datenbank des US-Medicare-Systems, in der Aufnahmediagnosen von Krankenhäusern sowie verordnete Medikamente erfasst werden. In dieser Studie wurden Patienten identifiziert, bei denen zwischen Oktober 2010

Graham DJ, Reichman ME, Wernecke $\mathrm{M}$ et al. Cardiovascular, bleeding, and mortality risks in elderly medicare patients treated with dabigatran or warfarin for non-valvular atrial fibrillation. Circulation 2014; Oct 30. pii: CIRCULATIO-NAHA.114.012061 und Dezember 2012 neu aufgetretenes Vorhofflimmern diagnostiziert wurde. Die Patienten wurden dann entweder mit Dabigatran oder mit Warfarin behandelt. In der Folgezeit wurden ischämische Insulte, intrakranielle Blutungen, schwerwiegende gastrointestinale Blutungen, akuter Myokardinfarkt und Tod erfasst. Für die statistische Auswertung erfolgte ein sogenanntes Propensity Score-Matching, das heißt die Patienten wurden jeweils bezüglich Risikofaktoren und Begleiterkrankungen vergleichbar gruppiert und dann ausgewertet.

Ergebnisse: Für diese Publikation lagen Daten von 134.414 Patienten vor, die entweder Dabigatran oder Warfarin erhielten. Für 67.207 Patienten, die neu Dabigatran bekamen, konnten entsprechende Patienten aus der Warfarin-Gruppe gematcht werden. Der überwiegende Anteil der Dabigatran-Patienten wurde mit der hohen Dosis von $2 \times 150$ mg behandelt. $15 \%$ erhielten die nur in den USA zugelassene niedrige Dosis von $2 \times 75 \mathrm{mg}$ täglich. Die meisten Patienten hatten einen CHADS-Score von 2 und einen HAS-BLED-Score von ebenfalls 2.

Die Auswertung zeigte eine 20 \%ige Risikoreduktion zugunsten von Dabigatran für ischämische Insulte und keinen Unterschied für schwerwiegende Blutungen. Das Risiko für gastrointestinale Blutungen war um $28 \%$ erhöht und das Risiko für intrakranielle und intrazerebrale Blutungen um $75 \%$ reduziert. Die Myokardinfarktraten waren identisch. Darüber hinaus ergab sich eine $14 \%$ ige signifikante Reduktion der Sterblichkeit zugunsten von Dabigatran. Bei der niedrigen Dosis von Dabigatran zeigten sich keine signifikanten Unterschiede gegenüber Warfarin für ischämischen Schlaganfall, schwerwiegende gastrointestinale Blutungen und Sterblichkeit. Die intrakraniellen Blutungen waren ebenfalls signifikant reduziert.

Schlussfolgerungen: Im klinischen Alltag lässt sich die in der RE-LY-Studie gezeigte Überlegenheit von Dabigatran im Vergleich zu Warfarin replizieren. Ischämische Insulte und intrakranielle Blutungen sind signifikant reduziert, das Risiko schwerwiegender gastrointestinaler Blutungen aber erhöht.

\section{- Kommentar von Hans-Christoph Diener, Essen}

\section{Die Alltagsdaten bestätigen die Ergebnisse der RE-LY-Studie}

Die vorliegende Analyse ist die bisher größte Beobachtungsstudie zum Vergleich von Dabigatran in überwiegend hoher Dosierung und Warfarin bei über 65-Jährigen. Die Methodik ist sehr ausgefeilt, da die beiden verglichenen Populationen über das Propensity Score-Matching vergleichbar sind. Wie in der RE-LY-Studie zeigt sich eine signifikante Reduktion von Schlaganfällen sowie ischämischen Insulten sowie eine vergleichbare Reduktion von intrakraniellen Blutungen und parenchymatösen Blutungen. Im Gegensatz zur RE-LY-Studie ergeben sich keinerlei Hinweise auf eine erhöhte Myokardinfarktrate. Die Mortalität war in der RE-LY-Studie ebenfalls reduziert, verpasste allerdings ganz knapp das Signifikanzniveau.

Ebenfalls wie in der RE-LY-Studie zeigt sich für die hohe Dosis von Dabigatran ein erhöhtes Risiko gastrointestinaler Blutun- gen. Dieses erhöhte Blutungsrisiko besteht nicht, wenn die nur in den USA zugelassene niedrige Dosis von $2 \times 75$ mg betrachtet wird. Diese Dosis wird bei Patienten mit deutlich eingeschränkter Nierenfunktion eingesetzt.

Leider ist in den USA die Dosis von $2 \times 110 \mathrm{mg}$ Dabigatran nicht zugelassen, sodass hierfür keine Daten vorliegen. Im klinischen Alltag wird allerdings empfohlen, bei Patienten im Alter über 75 Jahren und einer Kreatinin-Clearance von $30-50 \mathrm{ml} /$ Minute $2 \times 110 \mathrm{mg}$ Dabigatran einzusetzen. Ebenso sollte die niedrige Dosis bei Patienten Verwendung finden, bei denen in der Vorgeschichte bereits eine gastrointestinale Blutung aufgetreten ist.

\footnotetext{
Referenz:

1. Connolly SJ et al. N Engl J Med 2009; 361: 1139-51
} 